\title{
Automated positioning dual-axis solar tracking system with precision elevation and azimuth angle control
}

\begin{abstract}
This paper presents a study on an automated positioning open-loop dual-axis solar tracking system. The solar tracker was designed and fabricated using standard cylindrical aluminium hollow and Polyuthrene (PE). The control system of the solar tracker was governed by Micro Controller Unit (MCU) with auxiliary devices which includes encoder and Global Positioning System (GPS). The sun path trajectory algorithm utilizing the astronomical equation and GPS information was also embedded in the system. The power generation performance of the dual-axis solar tracking system was compared with the fixed-tilted Photovoltaic (PV) system. It is found that the solar tracker is able to position itself automatically based on sun path trajectory algorithm with an accuracy of $\pm 0.5^{\circ}$. The embedded Proportional Integral Derivative (PID) positioning system improves the tracking of elevation and azimuth angles with minimum energy consumption. It is reveals that the proposed solar tracker is able generate $26.9 \%$ and $12.8 \%$ higher power than fixed-tilted PV system on a clear and heavy overcast conditions respectively. Overall, the open-loop dual-axis solar tracker can be deployed automatically at any location on the earth with minimal configurations and is suitable for mobile solar tracking system.
\end{abstract}

Keyword: Automated positioning; Dual-axis solar tracker; PV; Elevation and azimuth angle control 